\title{
Neuromuscular Control of the Face-Head-Neck Biomechanical Complex With Learning-Based Expression Transfer From Images and Videos
}

\author{
XIAO S. ZENG*, University of California, Los Angeles, USA \\ SURYA DWARAKANATH* ${ }^{*}$, University of California, Los Angeles, USA \\ WUYUE LU, University of California, Los Angeles, USA \\ MASAKI NAKADA, NeuralX, Inc., Los Angeles, USA \\ DEMETRI TERZOPOULOS, University of California, Los Angeles, USA
}
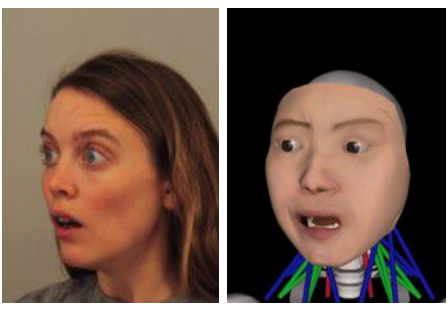

(a) Subject 1

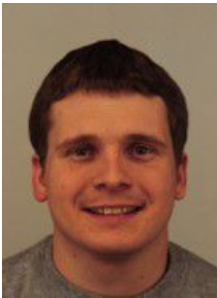

(b) Subject 2
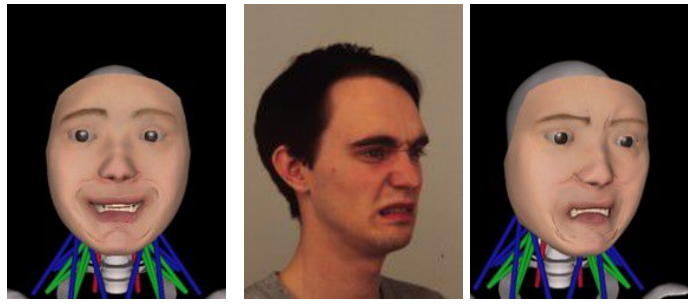

(c) Subject 3

Fig. 1. Our deep learning framework enables the transfer of facial expressions and head poses from images or videos to a biomechanical face-head-neck model.

The transfer of facial expressions from people to 3D face models is a classic computer graphics problem. In this paper, we present a novel, learning-based approach to transferring facial expressions and head movements from images and videos to a biomechanical model of the face-head-neck complex. Leveraging the Facial Action Coding System (FACS) as an intermediate representation of the expression space, we train a deep neural network to take in FACS Action Units (AUs) and output suitable facial muscle and jaw activation signals for the musculoskeletal model. Through biomechanical simulation, the activations deform the facial soft tissues, thereby transferring the expression to the model. Our approach has advantages over previous approaches. First, the facial expressions are anatomically consistent as our biomechanical model emulates the relevant anatomy of the face, head, and neck. Second, by training the neural network using data generated from the biomechanical model itself, we eliminate the manual effort of data collection for expression transfer. The success of our approach is demonstrated through experiments involving the transfer onto our face-head-neck model of facial expressions and head poses from a range of facial images and videos.

CCS Concepts: • Computing methodologies $\rightarrow$ Computer graphics; Animation; Neural networks.

Additional Key Words and Phrases: Biomechanical Human Animation, Facial Animation, FACS, Facial Expression Transfer, Neural Networks, Deep Learning, Computer Graphics, Computer Vision

\section{INTRODUCTION}

The face, actuated by the muscles of facial expression, and head movements, actuated by the cervical muscles, are a powerful mode of nonverbal communication between humans. The simulation of the face-head-neck musculoskeletal complex is of importance in understanding how we convey thinking and feeling in fields from affective science to 3D computer animation. Biomechanical human musculoskeletal models realistically capture the anatomy and physics underlying human motion generation. In particular, those pertaining to the simulation of the human face have succeeded in

\footnotetext{
${ }^{*}$ Co-first author.
} 


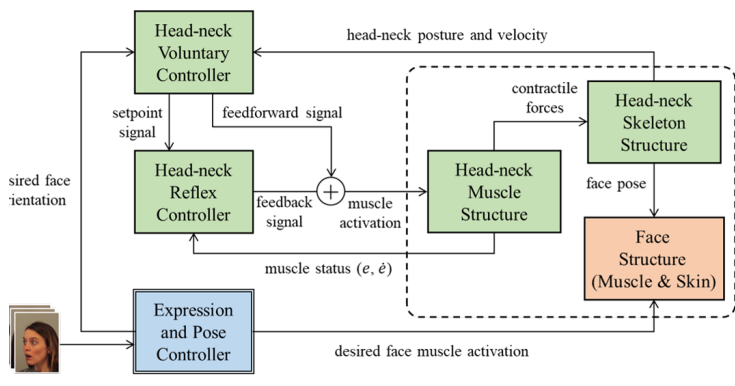

(a) Framework
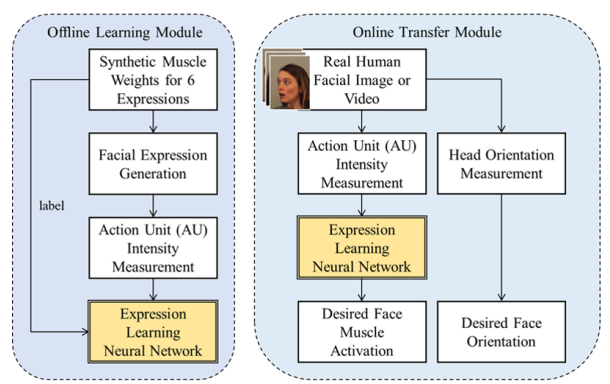

(b) Controller

Fig. 2. The structure of our expression and head pose transfer framework (a) and the components of the facial expression and head pose controller (b). The expression learning neural network (yellow) is first trained offline. In a transfer task, an image or a video sequence of a real face is fed into the online transfer module of the expression and pose controller to output the desired facial muscle activations and head orientation information. The muscle activations are input to the biomechanical face model (orange) to perform the corresponding expression and the head orientation is provided to the head-neck biomechanical neuromuscular system (green) to produce the desired head pose.

convincingly emulating facial expressiveness; however, they require significant effort in parameter tuning to produce realistic results.

In this paper, we show how to endow a biomechanical musculoskeletal model of the human face with the ability to produce facial expressions via machine learning from real-world reference images and videos (Figure 1). To this end, we introduce a deep neural-network-based method for learning the representation of human facial expressions through Ekman's Facial Action Coding System (FACS) [Cohn et al. 2007] in the context of the muscle actuators that drive the musculoskeletal face model augmented with a musculoskeletal cervicocephalic (neck-head) system to animate head movement during facial expression synthesis.

The novelty of our framework lies in the following features:

(1) We propose the first biomechanical face-head-neck animation system that is capable of learning to reproduce expressions and head orientations through neuromuscular control.

(2) Our novel deep neuromuscular motor controller learns to map between FACS Action Units (AUs) extracted from human facial images and videos and the activations of the muscle actuators that drive the biomechanical system.

(3) As a proof of concept, we demonstrate an automated processing pipeline (Figure 2) for animating expressions and head poses using an improved version of the physics-based neckhead-face animation system developed by Lee and Terzopoulos [Lee and Terzopoulos 2006], but which can potentially be applied to any muscle-driven model.

\section{RELATED WORK}

Muscle-based facial animation has been studied for decades [Waters 1987]. A series of studies have endeavored to build increasingly anatomically accurate musculoskeletal face models. Using such models, Terzopoulos and Waters [1993] and Sifakis et al. [2005], among others, have addressed the automatic determination of facial muscle activations from videos, but these methods require nonrigid facial motion trackers or motion capture markers. In this context, the work by Ishikawa et al. [1998, 2000] is of particular interest in that it employed three trained neural networks to transduce from the positions of facial markers to the activations of facial muscles. We present a 
markerless, real-time biomechanical face-head-neck simulation system that can automatically learn muscle activation parameters for a variety of expressions from reference images and videos. Most relevant to our work in the present paper is an existing biomechanical model of the cervicocephalic complex [Lee and Terzopoulos 2006], which incorporates an improved version of the face model developed by Lee et al. [1995].

Many facial animation researchers have made use of the well-known FACS [Ekman and Friesen 1978], a quantitative phenomenological abstraction that decomposes facial expressions into the intensity levels of Action Units (AUs), each of which corresponds to the actions of one or more facial muscles. An advantage of using the FACS is that it encodes anatomical and psychological semantics [Cohn et al. 2007].

Expression transfer or retargeting is a trending topic and recent approaches often use FACSbased blendshapes as the basic parametric representation [Thies et al. 2016; Weise et al. 2011; Zhang et al. 2020]. Others have used techniques such as interactive mesh deformation control [Xu et al. 2014] and neural-network-based perceptual models [Aneja et al. 2016] to represent expressions in blendshapes. However, transferring expressions using a musculoskeletal system is more natural since facial actions are the result of coordinated muscle contractions inducing soft tissue deformation. Despite the existing literature, there remains a deficiency of work in transferring expressions to musculoskeletal facial systems.

\section{MUSCULOSKELETAL MODEL}

Our real-time musculoskeletal model is based on the one of Lee and Terzopoulos [2006], but both the underlying face-head-neck control system and the facial expression system are significantly improved. Its overall architecture is controlled in a hierarchical manner, as illustrated in Figure $2 \mathrm{a}$. Specifically, the skeletal structure is an articulated multibody dynamics system, with bones and joints consistent with human anatomy. The skeletal system is driven by a Hill-type muscle actuator model.

The biomechanical face component consists of a facial soft tissue model comprising epidermis, dermal-fatty, fascia, and muscle layers supported by an underlying skull, which is constructed based on the work of Lee et al. [1995]. The soft tissue is a deformable model assembled from discrete uniaxial finite elements, which simulates dynamic facial deformation in an anatomically consistent yet simplified way compared to the models described in [Sifakis et al. 2005], [Wu et al. 2014], and [Cong et al. 2015], thus maintaining a low computational cost that affords real-time simulation performance on readily available hardware.

There are 26 pairs of primary facial muscles embedded in the biomechanical face, including the frontalis, corrugator, levator labii, orbicularis oculi, mentalis, and orbicularis oris muscle groups. The contractions of these muscles apply forces to the facial soft tissue layers, inducing deformations that produce meaningful facial expressions. We have augmented the expressive details, such as wrinkles on the face model, by applying multiple levels of subdivision to increase significantly the number of surface nodes that can be activated by muscle forces, and applied a high resolution texture map to the surface mesh for a natural appearance.

Appendix A explains the biomechanical components of our model in greater detail.

\subsection{Control}

To control the face-head-neck system, our novel neural network-based expression and pose controller generates facial muscle activations that produce recognizable expressions. It concurrently outputs head pose estimates to the head-neck musculoskeletal complex, where voluntary and reflex neuromuscular control layers generate muscle activation signals to achieve the desired head orientations. 


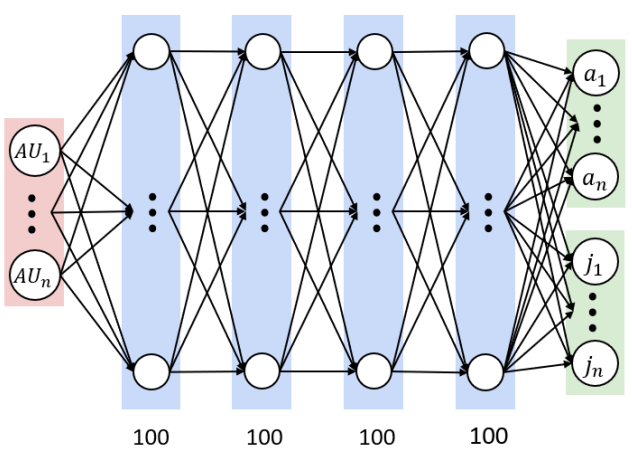

(a) Neural network architecture

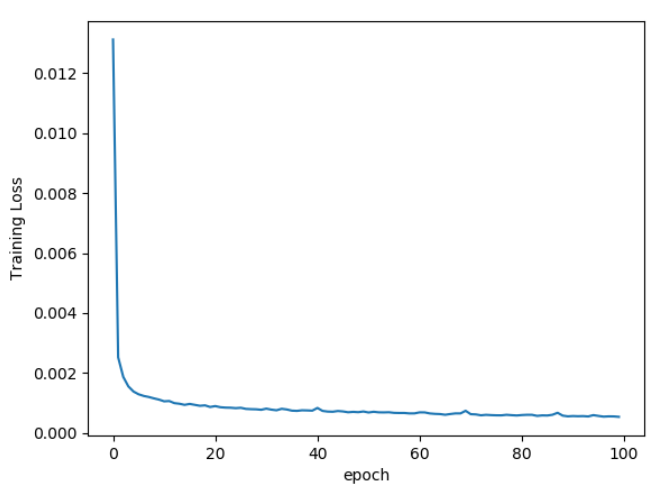

(b) Convergence of the neural network

Fig. 3. The expression learning neural network.

The higher-level voluntary controller receives the current head-neck posture and velocity information, as well as the desired adjustment of the posture, and generates a feedforward muscle activation signal and a setpoint control signal. The latter, which encodes the desired muscle strains and strain rates, is input to the reflex controller that then generates a feedback muscle activation signal and adds it to the feedforward signal generated by the voluntary controller. As a result, each cervical muscle receives an activation signal $a$ and, through simulation, generates a contractile muscle force accordingly. Together with the external environmental forces and gravity, the whole system is simulated through time and rendered as a physics-based animation. The voluntary controller runs at $25 \mathrm{~Hz}$ (in simulation time), while the reflex controller runs at $4 \mathrm{KHz}$ along with the physical simulation time steps.

\section{EXPRESSION LEARNING}

We next explain our machine learning approach of using a deep neural network to transfer facial expressions to our biomechanical face model. We leverage the FACS and synthesize the muscle activations for the model using the trained deep neural network. The following sections describe the architecture of the network, the pipeline for using the biomechanical face model to generate training data, and the process of training the neural network.

\subsection{Network Architecture}

The function of the neural network is to generate activation signals for the muscles of the biomechanical face model such that they generate contractile forces that deform the synthetic facial tissues to produce facial expressions. We employ a fully connected deep neural network architecture for this purpose (Figure 3a). The input layer consists of a total of 17 neurons representing the important AUs that are involved in the majority of facial movements, each neuron corresponding to a single normalized AU. We include 4 hidden layers with 100 neurons in each layer and ReLU activation. ${ }^{1}$ The output layer consists of 56 neurons, 52 of which encode the activations $a_{i}$, with $1 \leq i \leq 52$, for each of the 26 pairs of facial muscles, and the remaining 4 encode the jaw rotation, jaw slide, jaw twist, and an auxiliary value. Given its architecture, the network has a total of 37,300 weights. It is implemented in Keras with a TensorFlow backend.

\footnotetext{
${ }^{1}$ This choice was based on observed Mean Squared Errors (MSE) on test data of networks with different numbers of hidden layers and different numbers of neurons per hidden layer.
} 


\subsection{Training Data Generation}

The training data generation process is divided into two steps:

(1) generation of muscle activations, and

(2) generation of AUs for the corresponding muscle activations.

Each basic expression requires a combination of muscles to be activated. Given $n$ muscles, we define $W_{e}$ as a set of weights $w_{i}$, with $1 \leq i \leq n$, which determine the effect each muscle will have on an expression $e$. The activation for each muscle $a_{i}$ for an expression is then defined as $a_{i}=w_{i} s$, where $w_{i} \in W_{e}$ and $s$ is a scale term. For a single expression, we determine the weights in a set $W_{e}$ manually by visually analyzing the facial expressions formed by the face model. We repeat the process for all the basic expressions, namely (1) Joy, (2) Sadness, (3) Anger, (4) Fear, (5) Disgust, and (6) Surprise to generate the sets $W_{\text {Joy }}, W_{\text {Sadness }}, W_{\text {Anger }}, W_{\text {Fear }}, W_{\text {Disgust }}$ and $W_{\text {Surprise }}$, respectively.

We then sample the value of $s$ randomly in the range $[0.0,1.0]$ to generate all the muscle activations $a_{i}$. We also assign a random value between 0 and 1 for the jaw rotation. For the purpose of our experiments, we maintain the jaw twist and jaw slide at a value of 0.5 . We further form a set $A$ consisting of all the muscle activations $a_{i}$, where $1 \leq i \leq n$, and the jaw activations including jaw rotation, jaw twist and jaw slide. We iterate the above process, generating the set $A$ by repeatedly sampling the value of $s$ for a single expression. We finally extend this to all basic expressions and obtain multiple sets $A_{\text {Joy }}, A_{\text {Sadness }}, A_{\text {Anger }}, A_{\text {Fear }}, A_{\text {Disgust }}$ and $A_{\text {Surprise }}$ for each expression.

We leverage the functionality of OpenFace 2.0 [Baltrusaitis et al. 2018] for facial expression recognition and head pose estimation. OpenFace is a computer vision tool capable of facial landmark detection, head pose estimation, facial AU recognition, and eye-gaze estimation. OpenFace employs Convolutional Experts Constrained Local Models (CE-CLMs) for facial landmark detection and tracking. CE-CLMs use a 3D representation of the detected facial landmarks by which OpenFace estimates the head pose. Eye gaze estimation is done using a Constrained Local Neural Fields (CLNF) landmark detector. The final task, facial expression recognition, is performed using Support Vector Machines and Support Vector Regression.

We use a single set $A$ formed by the above described procedure to activate the muscles and jaw of the biomechanical face model. We then render the model to form an image. The image is input to OpenFace, which performs facial expression recognition and head pose estimation, outputting the estimated AUs and head orientation associated with the input image. We repeat this process for each set $A$ formed (as described previously) to obtain the corresponding AUs and head orientations.

Thus, we synthesize a large quantity of training data pairs each consisting of (i) muscle and jaw activations $A$ and (ii) the associated AUs and head orientations.

\subsection{Network Training}

We use the aforementioned data to train our deep neural network to input AUs and output corresponding muscle and jaw activations.

The AUs from each training pair, generated as described in the previous section, are passed to the network as input. We then compare the corresponding muscle and jaw activations; i.e., the ground truth compared to the predictions of muscle and jaw activations given by the neural network. We use a Mean Square Error (MSE) training loss between the predictions and the ground truth, which is backpropagated to update the weights, thus training the neural network.

We normalize the intensity values of each AU class across all pairs to remove the disparity of intensity values between synthetic faces and real faces. We use a total of 6,000 pairs, with about 1,000 pairs for each basic expression.

To train the neural network, we use the ADAM stochastic gradient descent optimizer with an MSE loss, a batch size of 32, and a learning rate of 0.01 . We train the network in a total of 100 
epochs, running on an NVIDIA GeForce GTX 1650 GPU installed on a Windows 10 machine with a 2.6GHz Intel Core i7-9750H CPU. Figure 3b shows the convergence of the training error.

\subsection{Expression Transfer Pipeline}

To transfer real facial expressions on the fly, we use a pipeline similar to the offline training module, again leveraging OpenFace for facial expression recognition and head pose estimation. We input an image of an expressive face into OpenFace to obtain all of the corresponding AUs and head orientations. The AUs are then passed into the trained neural network which outputs predictions of the muscle and jaw activations, driving the biomechanical face to deform the muscles and transfer the expressions onto it.

We transfer both image and video inputs. Each frame in a video is processed independently and a resulting video is created using the transferred frames. The intensity values for each AU class are normalized across all the images or frames as in the case of the training pipeline.

\section{EXPERIMENTS AND RESULTS}

We next present the results of transferring facial expressions from the wild using our trained neural network. We evaluate our expression transfer pipeline on different expressions while using a variation of AUs and muscles in the biomechanical face model.

\subsection{Facial Expression Datasets}

Several facial expression datasets are available online. The datasets that we used in our experiments are as follows:

Karolinska Directed Emotional Faces (KDEF) [Calvo and Lundqvist 2008]. The KDEF dataset consists of 4,900 pictures of human facial expressions. It covers 70 subjects ( 35 female and 35 male) enacting all the basic facial expressions, namely Neutral, Joy, Sadness, Anger, Fear, Disgust, and Surprise. Each expression performed by the subject is imaged from multiple directions. We use the dataset to transfer facial expressions onto the biomechanical face model and visually analyze the performance of our trained neural network in this paper.

Cohn Kanade Dataset (CK) and Extended Cohn Kanade Dataset (CK+) [Kanade et al. 2000; Lucey et al. 2010]. The $\mathrm{CK}$ and the $\mathrm{CK}+$ dataset combined consist of 593 video sequences of 123 subjects. Each sequence consists of images from a neutral expression (first frame) to a peak expression (last frame). The peak expressions are FACS coded for AUs. We use the sequences in the CK+ dataset to transfer videos of expression transitions onto the biomechanical face.

Ryerson Audio-Visual Database of Emotional Speech and Song (RAVDESS) [Livingstone and Russo 2018]. The original RAVDESS dataset consists of 24 actors vocalizing two lexically-matched statements. An extension of the dataset, named RAVDESS Facial Landmark Tracking, contains tracked facial landmark movements from the original RAVDESS datasets [Swanson et al. 2019]. Each actor performs 60 speech trials and about 44 song trials. This yields a total of 2,452 video files for the complete dataset. We leverage this dataset to test the transfer of actor faces in speech videos onto the biomechanical face.

\subsection{Action Units and Muscle Activations}

There exist a total of 30 Action Units (AUs) corresponding to facial expressions. OpenFace provides occurrence predictions for 18 out the 30 AUs and measures intensity values for 17 out of the 30 AUs. We consider the 17 AUs for which the intensity values are present as the super-set of the AUs for our use case.

Due to the correlation between the AUs and muscles in the face, there also exists a correlation between a basic facial expression and the AUs activated by it. Our initial experiments focused on 
Table 1. Comparison of the Mean Squared Errors for training individual neural networks for each expression and for training a single neural network for all expressions.

\begin{tabular}{ccccccc}
\hline MSE $_{\text {Joy }}$ & MSE $_{\text {Sadness }}$ & MSE $_{\text {Fear }}$ & MSE $_{\text {Anger }}$ & MSE $_{\text {Surprise }}$ & MSE $_{\text {Disgust }}$ & MSE $_{\text {All }}$ \\
\hline 0.000591 & 0.002925 & 0.001611 & 0.009689 & 0.000266 & 0.002516 & 0.000729 \\
\hline
\end{tabular}

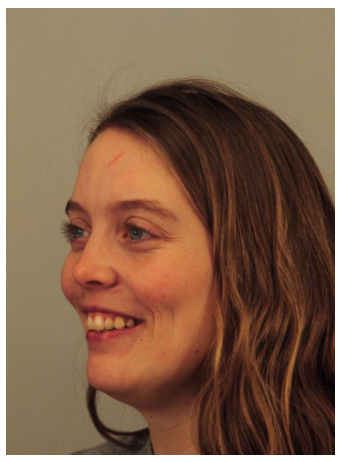

(a) Subject 1

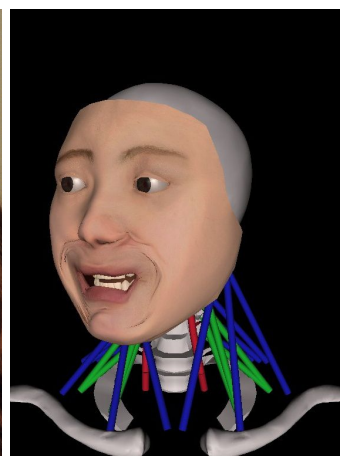

Fig. 4. Transfer of head orientation along with facial expression.

training the neural network for each expression in an isolated manner. The neural network was trained to output muscle activation for muscles corresponding to a single expression using AUs which pertained to the same expression. In further trials, we observed that usage of all 17 AUs and all facial muscles improved the performance and the scalability of the expression transfer pipeline.

Table. 1 provides a comparison between training a single neural network for all expressions and training individual neural networks for each expression, where each network is trained only from the AUs and muscles relevant to its expression (using the mappings of expressions to AUs and AUs to muscles presented in [Du et al. 2014], [Clark et al. 2020] and [Scherer et al. 2019]). We observe better performance when training a single neural network for all the expressions, suggesting that AUs not directly relevant to an expression also play a role in expression transfer.

Due to limitations in the biomechanical model, the range of each AU class differs from that of the real faces. Hence, we use normalization to overcome the bias and better transfer real facial expressions onto the biomechanical model. The expression intensities in transferred expressions with normalization better represent the real faces than those without normalization.

We choose to activate only jaw rotation so as to maintain the symmetry of the expression for our use case. We observe, that without jaw activations, expressions such as surprise are not well synthesized by the biomechanical face model.

\subsection{Head Orientation}

Leveraging OpenFace for head pose estimation, we pass the estimated orientation of the head into the trained neck controller to activate the neck muscles. This in turn actuates the neck to adjust the head orientation in accordance with the input image. Figure 4 presents sample transferred results including head orientations from the KDEF dataset. 

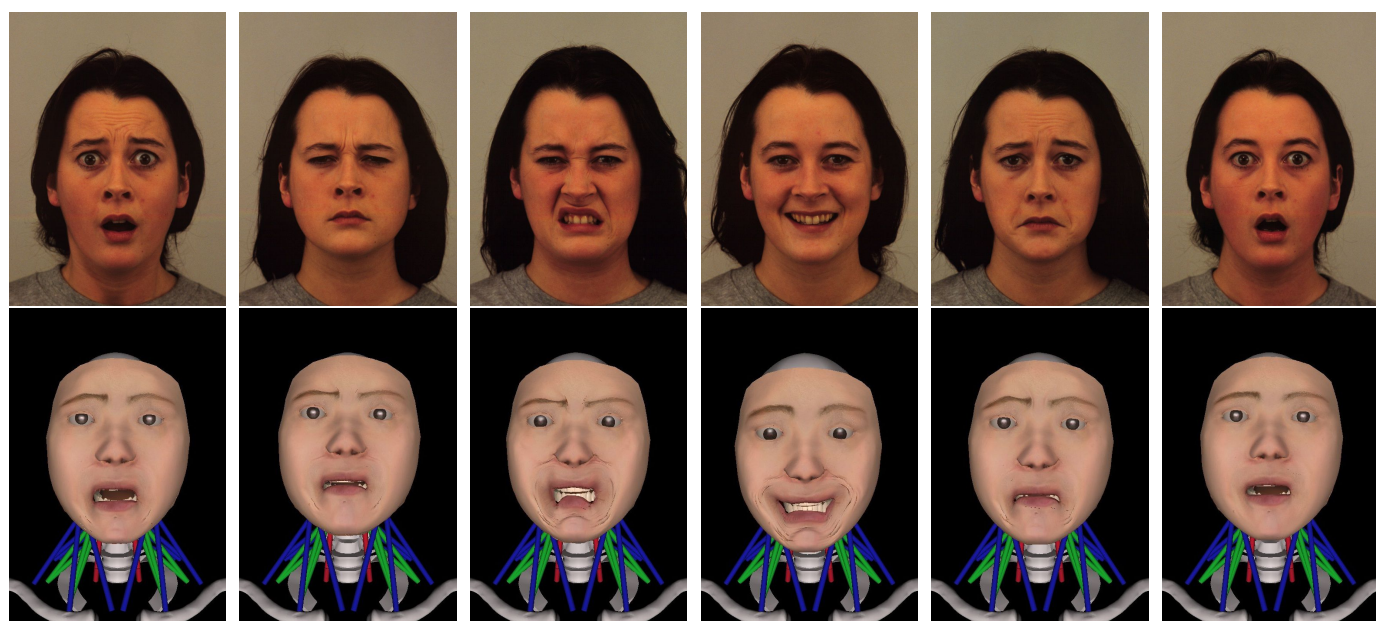

(a) Subject 4
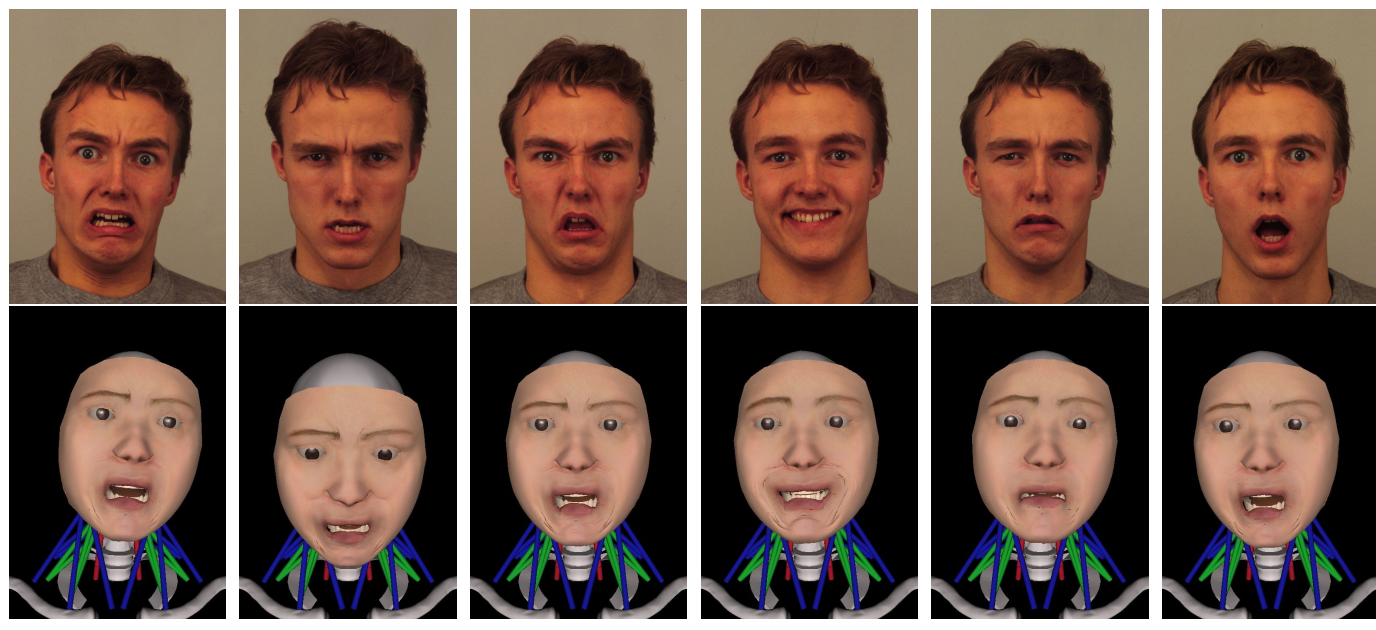

(b) Subject 5

Fig. 5. Transfer of facial expressions (fear, anger, disgust, joy, sadness, surprise) and head poses from KDEF images to the biomechanical face-head-neck model.

\subsection{Facial Expression Transfer}

The results for the facial expression transfer for each of the expressions is shown in Figure 5. We present transfer results of a small sample of the KDEF dataset, selecting two subjects (a female and a male) enacting all the basic expressions.

We also evaluate the transfer results by comparing the MSE of selected AUs in Table 2. We calculate the average MSE by AUs over all transferred expressions and their corresponding reference data, then compute the mean value of the average MSEs over all AUs in each experimental setting. We report such mean values together with 8 randomly chosen AUs. The normalization step decreases the MSEs of most selected AUs and yields results with higher AU similarity.

Figure 6 shows selected frames of the transfer of expressions from a video in the RAVDESS dataset (best seen in our supplemental video). The more subtle expressions performed by subjects 
Table 2. Average MSE of selected AUs in the original data and transfer results using different settings. Note that we randomly select 8 out of 17 AUs output by OpenFace. The last column is the average MSE over all the AUs in each setting.

\begin{tabular}{lccccccccc}
\hline Dataset & MSE $_{\mathrm{AU} 1}$ & MSE $_{\mathrm{AU} 4}$ & MSE $_{\mathrm{AU} 6}$ & MSE $_{\mathrm{AU} 9}$ & MSE $_{\mathrm{AU} 12}$ & MSE $_{\mathrm{AU} 15}$ & MSE $_{\mathrm{AU} 20}$ & MSE $_{\mathrm{AU} 25}$ & Average \\
\hline KDEF (unnormalized) & 0.191 & 0.088 & 0.186 & 0.133 & 0.011 & 0.155 & 0.129 & 0.092 & 0.157 \\
KDEF (normalized) & 0.118 & 0.064 & 0.186 & 0.068 & 0.028 & 0.109 & 0.146 & 0.052 & 0.129 \\
RAVDESS (video) & 0.012 & 0.222 & 0.107 & 0.044 & 0.083 & 0.016 & 0.077 & 0.036 & 0.086 \\
CK (video) & 0.038 & 0.054 & 0.059 & 0.015 & 0.008 & 0.023 & 0.057 & 0.034 & 0.048 \\
\hline
\end{tabular}

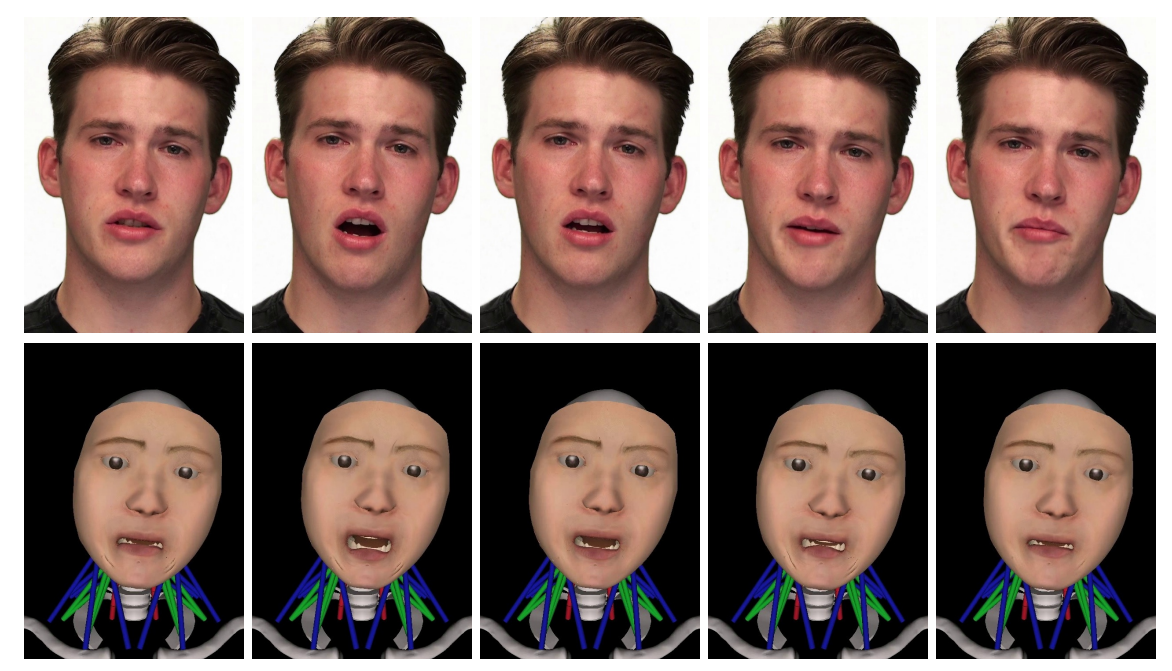

Fig. 6. Facial expression and head pose transfer from a video image sequence from the RAVDESS dataset of a talking subject expressing Sadness.

in the RAVDESS and CK videos result in lower average MSEs compared with those for the KDEF image dataset.

\section{CONCLUSIONS AND FUTURE WORK}

Our expression transfer approach is uniquely advantageous. First, it is anatomically consistent as the biomechanical model emulates the human cervicocephalic musculoskeletal system. Further, our approach is based on the Facial Action Coding System, which is a widely adopted representation of facial expressions, including in the computer animation field. Finally, our approach is based on deep learning, which can capture the complex, non-linear relation between the Action Units of the FACS and associated facial muscle activations.

Additionally, our neural-network-based approach does not require any manual data collection as the training data is generated directly using the biomechanical model itself. This only needs to take place once, in advance. Once trained offline using the synthesized training data, the neural network can quickly transfer a large number of facial expressions. Unlike popular face tracking software such as Faceware, which requires manual calibration steps to transfer expressions of different subjects, our proposed approach needs no additional parameter adjustments to perform the same task. 
Although we have achieved satisfactory transfer results for all the basic facial expressions, note that the simulation and transfer of complex mouth and eye movements lies outside the scope of our current work. To improve the fidelity of the results, we plan to extend our musculoskeletal model (e.g., by adding more muscles to help activate facial AUs in a more anatomically accurate manner) and its associated control system so as to explicitly control the lips and eyes, as well as to transfer mixtures of expressions, subtle expressions, and microexpressions, which the human face is capable of producing.

\section{ACKNOWLEDGMENTS}

We thank CyberAgent, Inc., for providing the high resolution texture for the facial mesh that we used in our model. We also thank the curators of RAVDESS, CK/CK+, and KDEF for granting us permission to use their datasets.

\section{A ADDITIONAL DETAILS OF THE BIOMECHANICAL MODEL}

\section{A.1 Skeleton}

The skeletal structure of our model contains 9 links and is modeled as an articulated rigid-body system. The bone links are built on the base link, which is set to be immobile. Among the 8 movable bones, there are seven cervical bones, named $\mathrm{C} 1$ to $\mathrm{C} 7$, and the skull. The human neck skeleton system contains soft tissue between vertebrae and the cervical spine, which enables motion with 6 degrees of freedom ( 3 for translation and 3 for rotation) for the bones. We simplify each bone joint to have just 3 rotational degrees of freedom.

The equation of motion of the rigid-body system can be written as

$$
\mathrm{M}(\mathrm{q}) \ddot{\mathrm{q}}+\mathrm{C}(\mathrm{q}, \dot{\mathrm{q}})+\mathrm{K}_{s} \mathrm{q}+\mathrm{K}_{d} \dot{\mathrm{q}}=\mathrm{P}(\mathrm{q}) \mathrm{f}_{m}+\mathrm{J}(\mathrm{q})^{\top} \mathrm{f}_{\text {ext }},
$$

where $\mathrm{q}, \dot{\mathrm{q}}$, and $\ddot{\mathrm{q}}$ are 24-dimensional vectors representing the joint angles, the angular velocities, and the angular accelerations, respectively. $\mathrm{M}$ is the mass matrix, and $\mathrm{C}$ represents the internal forces among the system, including Coriolis forces, gravity, and forces from connecting tissues. The moment arm matrix $\mathrm{P}$ maps the muscle force $\mathrm{f}_{m}$ (contractile muscle force $\mathrm{f}_{C}$ and passive muscle force $\mathrm{f}_{P}$ ) to the related joint torques, while the Jacobian matrix $\mathrm{J}$ transforms the applied external force $\mathrm{f}_{\text {ext }}$ to torques. The $\mathrm{K}_{s} \mathrm{q}+\mathrm{K}_{d} \dot{\mathrm{q}}$ term represents the rotational damping springs that we attach to the joints in order to simulate the stiffness of the inter-vertebral discs. We can alternatively write the torque from the spring as:

$$
\tau_{s}=-k_{s}\left(q-q_{0}\right)-k_{d} \dot{q}
$$

where $q$ is the current joint angle in the generalized coordinates, and $q_{0}$ is the joint angle in the natural pose (resting angle). The $k_{s}$ and $k_{d}$ are the stiffness and damping coefficients of the spring, respectively.

\section{A.2 Muscles}

We use a modified version of the Hill-type muscle model [Ng-Thow-Hing 2001], which is a good balance of biomechanical accuracy and computational efficiency. The muscle force $f_{m}=f_{P}+f_{C}$ has two sources. The passive element $f_{P}$ generates a restoring force due to the muscle elasticity, which constrains the muscle deformation passively. The passive muscle force is represented as

$$
f_{P}=\max \left(0, k_{s}\left(\exp \left(k_{c} e\right)-1\right)+k_{d} \dot{e}\right),
$$

where $k_{s}$ and $k_{d}$ are the stiffness and damping coefficients of the above uni-axial exponential spring model. $e$ is the strain of the muscle and $\dot{e}$ is the strain rate. We can calculate them using $e=\left(l-l_{0}\right) / l_{0}$ and $\dot{e}=\dot{l} / l_{0}$, respectively, where $l$ and $l_{0}$ are the muscle length and muscle resting length. 


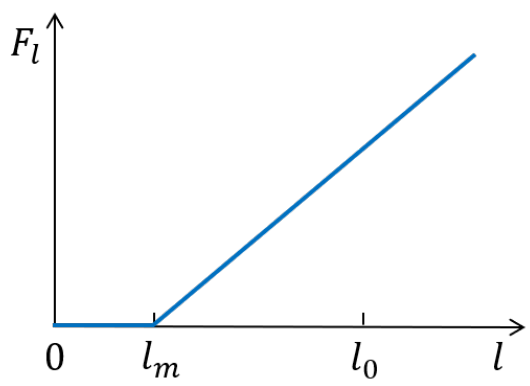

(a) Force-length relationship

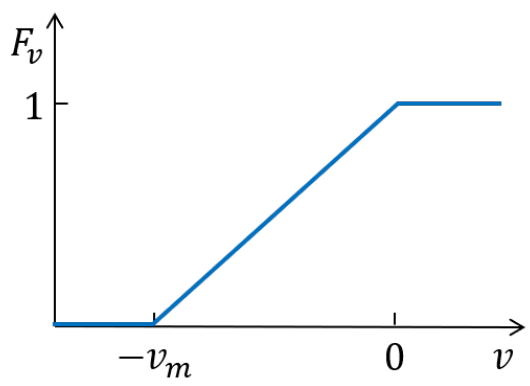

(b) Force-velocity relationship

Fig. 7. The force relationships of Hill type muscle model.

The contractile element $f_{C}$ generates the proactive contractile force of the muscle, which is proportionate to the activation level of the muscle:

$$
\begin{aligned}
f_{C} & =a F_{l}(l) F_{v}(i), \\
F_{l}(l) & =\max \left(0, k_{\max }\left(l-l_{m}\right)\right), \\
F_{v}(i) & =\max \left(0,1+\min (i, 0) / v_{m}\right),
\end{aligned}
$$

where $a \in[0,1]$ is the muscle activation level, $F_{l}$ is the force-length relation, and $F_{v}$ is the forcevelocity relation. During their calculation, we need the maximum muscle stiffness $k_{\text {max }}$, maximum muscle length $l_{m}$, and the maximum contractile velocity $v_{m}$. We present the plots of the two relations in Figure 7. Additional details about the setting and the biomechanical background of the parameters can be found in [Lee and Terzopoulos 2006].

\section{A.3 Skin}

Our facial skin model is automatically generated using the technique described in [Lee et al. 1995]. After laser-scanning the individual's facial data, a range image and a reflectance image in cylindrical coordinates are adapted to a well-structured face mesh. Then the algorithm creates a dynamic skin and muscle model for the face mesh, which contains automatically generated facial soft tissues, estimated skull surface. Also, major muscles responsible for facial expression are inserted to the model.

The physical simulation of the muscle-actuated facial skin model is implemented as a discrete deformable model (DDM), where a network of fascia nodes are connected using uni-axial springs. The force exerts from spring $j$ on node $i$ can be written as

$$
\mathrm{g}_{i}^{j}=c_{j}\left(l_{j}-l_{j}^{r}\right) \mathrm{s}_{j}
$$

where $l_{j}$ and $l_{j}^{r}$ are the current and resting length of spring $j$, and $\mathrm{s}_{j}=\left(\mathrm{x}_{j}-\mathrm{x}_{i}\right) / l_{j}$ is the spring direction vector.

The facial skin model is also actuated by the underlying muscles. We calculate the force exerts from muscle $j$ on node $i$ according to the length scaling function $\Theta_{1}$ and the muscle-width scaling function $\Theta_{2}$ as follows:

$$
f_{i}^{j}=\Theta_{1}\left(\varepsilon_{j, i}\right) \Theta_{2}\left(\omega_{j, i}\right) .
$$

In [Lee et al. 1995], the plots of the two scaling functions, as well as the definition of the length ratio $\varepsilon$ and muscle width $\omega$ are explained in detail. Moreover, there are other aspects of the generated 
discrete deformable model; e.g., the volume preservation forces and skull penetration constraint forces.

\section{REFERENCES}

Aneja, D., Colburn, A., Faigin, G., Shapiro, L., and Mones, B. (2016). Modeling stylized character expressions via deep learning. In Proc. Asian Conference on Computer Vision, pages 136-153.

Baltrusaitis, T., Zadeh, A., Lim, Y. C., and Morency, L.-P. (2018). OpenFace 2.0: Facial behavior analysis toolkit. In Proc. IEEE Conference on Automatic Face and Gesture Recognition, pages 59-66.

Calvo, M. G. and Lundqvist, D. (2008). Facial expressions of emotion (kdef): Identification under different display-duration conditions. Behavior Research Methods, 40(1):109-115.

Clark, E. A., Kessinger, J., Duncan, S. E., Bell, M. A., Lahne, J., Gallagher, D. L., and O’Keefe, S. F. (2020). The facial action coding system for characterization of human affective response to consumer product-based stimuli: A systematic review. Frontiers in Psychology, 11:920.

Cohn, J. F., Ambadar, Z., and Ekman, P. (2007). Observer-based measurement of facial expression with the facial action coding system. The Handbook of Emotion Elicitation and Assessment, 1(3):203-221.

Cong, M., Bao, M., E, J. L., Bhat, K. S., and Fedkiw, R. (2015). Fully automatic generation of anatomical face simulation models. In Proc. ACM SIGGRAPH/EG Symposium on Computer Animation, pages 175-183.

Du, S., Tao, Y., and Martinez, A. M. (2014). Compound facial expressions of emotion. Proceedings of the National Academy of Sciences, 111(15):E1454-E1462.

Ekman, P. and Friesen, W. V. (1978). Manual for the Facial Action Coding System. Consulting Psychologists Press.

Ishikawa, T., Morishima, S., and Terzopoulos, D. (1998). 3D estimation of facial muscle parameter from the 2D marker movement using neural network. In Proc. Third Asian Conference on Computer Vision, Lecture Notes in Computer Science, Vol 1352., pages 671-678. Springer.

Ishikawa, T., Morishima, S., and Terzopoulos, D. (2000). 3D face expression estimation and generation from 2D image based on a physically constraint model. IEICE Transactions on Information and Systems, 83(2):251-258.

Kanade, T., Cohn, J., and Tian, Y. (2000). Comprehensive database for facial expression analysis. In Proceedings of the Fourth IEEE International Conference on Automatic Face and Gesture Recognition, pages 46-53.

Lee, S.-H. and Terzopoulos, D. (2006). Heads up! Biomechanical modeling and neuromuscular control of the neck. ACM Transactions on Graphics, 25(3):1188-1198.

Lee, Y., Terzopoulos, D., and Waters, K. (1995). Realistic modeling for facial animation. In Proc. ACM SIGGRAPH 95 Conference, pages 55-62.

Livingstone, S. R. and Russo, F. A. (2018). The Ryerson audio-visual database of emotional speech and song (RAVDESS): A dynamic, multimodal set of facial and vocal expressions in North American English. PLOS ONE, 13(5):1-35.

Lucey, P., Cohn, J. F., Kanade, T., Saragih, J., Ambadar, Z., and Matthews, I. (2010). The extended Cohn-Kanade dataset (ck+): A complete dataset for action unit and emotion-specified expression. In Proc. IEEE CVPR Workshops, pages 94-101.

Ng-Thow-Hing, V. (2001). Anatomically-based models for physical and geometric reconstruction of humans and other animals. $\mathrm{PhD}$ thesis, Department of Computer Science, University of Toronto.

Scherer, K. R., Ellgring, H., Dieckmann, A., Unfried, M., and Mortillaro, M. (2019). Dynamic facial expression of emotion and observer inference. Frontiers in Psychology, 10:508.

Sifakis, E., Neverov, I., and Fedkiw, R. (2005). Automatic determination of facial muscle activations from sparse motion capture marker data. In Proc. ACM SIGGRAPH 2005 Conference, pages 417-425.

Swanson, R., Livingstone, S., and Russo, F. (2019). RAVDESS facial landmark tracking (version 1.0.0) [dataset].

Terzopoulos, D. and Waters, K. (1993). Analysis and synthesis of facial image sequences using physical and anatomical models. IEEE Transactions on Pattern Analysis and Machine Intelligence, 15(6):569-579.

Thies, J., Zollhofer, M., Stamminger, M., Theobalt, C., and Nießner, M. (2016). Face2face: Real-time face capture and reenactment of RGB videos. In Proc. IEEE Conference on Computer Vision and Pattern Recognition, pages 2387-2395.

Waters, K. (1987). A muscle model for animation three-dimensional facial expression. Proc. ACM SIGGRAPH 87 Conference, 21(4):17-24.

Weise, T., Bouaziz, S., Li, H., and Pauly, M. (2011). Realtime performance-based facial animation. ACM Transactions on Graphics, 30(4):1-10.

$\mathrm{Wu}$, T., Hung, A., and Mithraratne, K. (2014). Generating facial expressions using an anatomically accurate biomechanical model. IEEE Transactions on Visualization and Computer Graphics, 20(11):1519-1529.

Xu, F., Chai, J., Liu, Y., and Tong, X. (2014). Controllable high-fidelity facial performance transfer. ACM Transactions on Graphics, 33(4):1-11.

Zhang, J., Chen, K., and Zheng, J. (2020). Facial expression retargeting from human to avatar made easy. IEEE Transactions on Visualization and Computer Graphics. 